\title{
The Glenn Shunt Revisited, A Single Center Registry in Ain Shams University Cardiology Department
}

\author{
Mina Tewfik $^{1,2, *}$, Maiy El-Sayed ${ }^{1}$, Alaa Roushdy ${ }^{1}$, Soha Romeih ${ }^{2,3}$, Dina Ezzeldin ${ }^{1}$ and Hebatalla Attia ${ }^{1}$ \\ ${ }^{1}$ Cardiology Department, Ain Shams University, Cairo, Egypt \\ ${ }^{2}$ Prof. Magdy Yacoub Aswan Heart Centre, Aswan, Egypt \\ ${ }^{3}$ Cardiology Department, Tanta University, Tanta, Egypt \\ *Corresponding Author: Mina Tewfik. Email: minaaziz_89@yahoo.com
}

Received: 20 July 2021 Accepted: 08 September 2021

\begin{abstract}
Background: Bidirectional Glenn shunts have long been available as palliative procedures for patients with single ventricle physiology that is, patients unsuitable for biventricular repair. In our country they are performed at an older age than that recommended by the literature. So, we aim to assess post bidirectional Glenn shunt patients to detect the presence of any complications and to evaluate their functional capacity. Methods: This was a descriptive study that included all patients who underwent a bidirectional Glenn shunt and were referred for follow up in Ain shams university hospitals. History taking including NYHA class and physical activity, clinical examination, six-minutes-walk test, laboratory investigations, full echocardiographic assessment were done for all patients. Some patients needed invasive cardiac catheterization. Results: Our registry included 178 patients who underwent bidirectional Glenn procedure referred for follow up in Ain Shams university hospital from January 2019 till July 2020. The mean age of the registry was $18.7 \pm 8.26$ years (range between 5 and 37 years), 85 males (48\%) and 93 females (52\%). Regarding the basic anatomy, double outlet right ventricle with hypoplastic left ventricle was the commonest. Furthermore, our descriptive study confirmed many characteristic similarities between our patients and patients in developing countries. Our patients underwent bidirectional Glenn shunt at a median age of 6 years which is considered a relatively old age but similar to other studies that have been made in developing countries like Pakistan, India and Iran. There is a significant delay in the operation in Egyptian patients due to lack of patients' awareness, few numbers of primary health care facilities and high economic burden. Conclusion: Although the presence of slight systemic desaturation, our study demonstrated a satisfactory functional capacity among our patients; thus, bidirectional Glenn can be considered an acceptable definite univentricular repair in patients with late presentation.
\end{abstract}

\section{KEYWORDS}

Bidirectional Glenn shunt; single ventricle; univentricular repair

\section{Introduction}

Among the most frequently encountered causes of fetal mortality and morbidity are congenital heart diseases, with an incidence of $1 \%$ in newborns [1,2]. Specially in developing countries, the exact incidence is vague and usually the presentation is delayed; and the reasons include insufficient registries, 
asymptomatic nature of disease at early months of life and missed diagnosis. Single ventricular physiology, also known as univentricular heart, requires an experienced heart team including pediatric cardiologist and cardiothoracic surgeons, in order to reach the most suitable management plan for each case. The management plan entails series of palliative operations like Norwood procedure, modified Blalock-Taussig shunt (MBTS), MBTS/Pulmonary artery banding, bidirectional Glenn shunt (BDG), and then Fontan completion.

Staged procedures towards Fontan completion are preferred providing better results and less complications. However, the choice of suitable candidate is crucial and must follow the Ten Commandments for an ideal Fontan operation [3]. These commandments include age younger than 4 years, sinus rhythm, normal venous return, normal right atrial dimension and volume, mean pulmonary artery pressure not exceeding $15 \mathrm{mmHg}$, pulmonary vascular resistance less than 1 Wood unit $/ \mathrm{m}^{2}$, pulmonary artery-aorta ratio more than 0.75 , preserved systemic ventricle function, competent mitral valve and good anatomy of pulmonary arteries.

Most importantly, it remains critical that the resistance across the pulmonary capillary bed remains low. Excessive pulmonary vascular resistance is a clear contraindication to a Fontan completion because pulmonary vascular resistance provides the primary limitation to cardiac output in patients without a prepulmonary pump [4].

The BDG is still an essential step in univentricular heart repair; as it offers excellent palliation and increased pulmonary blood flow. In addition, it unloads the heart and allows pulmonary artery growth, hence, it improves survival and quality of life [5].

Although BDG is routinely used as a step towards Fontan procedure or a definite palliation in high-risk candidates for Fontan, little is known about the late results, the duration of palliation, and the frequency with which this procedure allows later successful conversion to a Fontan type of procedure. Therefore, in the current study, we aim to report present-day outcomes following BDG in patients with various single ventricle anomalies and to assess the post BDG patients' functional capacity and desaturation. The ultimate goal is to identify how satisfactory is our patients' quality of life and if BDG could be considered as a standard of care for univentricular repair in late presenter population.

\section{Materials and Methods}

This was a retrospective study including patients who underwent BDG referred for follow up in Ain Shams University hospitals from January 2019 till July 2020.

Inclusion criteria:

All patients who underwent BDG for follow up in Ain shams university hospital were included. Pediatric and adult patients were both included in the study.

Exclusion criteria:

Critical ill patients (patients with hemodynamic instability or significant neurological deficit) and patients refusing the study.

After the informed consent, all patients were subjected to the following:

\subsection{History}

Proper history taking by a customized questionnaire including: demographic data (gender, current age, weight, height, body surface area, order of birth), relevant family history (familial congenital heart disease, consanguinity and maternal risk factors), clinical data (age at first presentation, functional capacity, level of physical activity and any reported symptoms), surgical data (age and weight at time of the operation, date of operation, surgical procedures other than BDG such as a previous pulmonary artery banding or 
aortopulmonary shunt), history of percutaneous interventions (Pulmonary artery stenting or veno-venous collaterals closure) and current medical treatment.

\subsection{Clinical Examination}

Including general examination with special emphasis on general appearance, specific features, complexion, vital data, clubbing, weight, height, peripheral pulsations, oedema, hepatomegaly and jugular venous pressure examination.

\subsection{Six-Minutes-Walk Test}

The test was performed according to American Thoracic Society Standard [6]. The distance, blood oxygen saturation before test (rest SAT) and desaturation at the maximum effort (rest SAT-SAT 6'), initial and maximal heart rate (rest HR, peak HR) and the recovery time were analyzed.

\subsection{Routine Investigation}

12 lead surface electrocardiogram (ECG) and routine laboratory investigations including (complete blood picture, kidney function, liver function, electrolytes and coagulation profile) were performed for all patients in the study.

\subsection{Conventional 2D Echocardiography}

Full ECG gated echocardiographic study using echocardiography machine with phased array transducer of suitable frequency to perform sequential segmental analysis (Figs. 1 and 2).

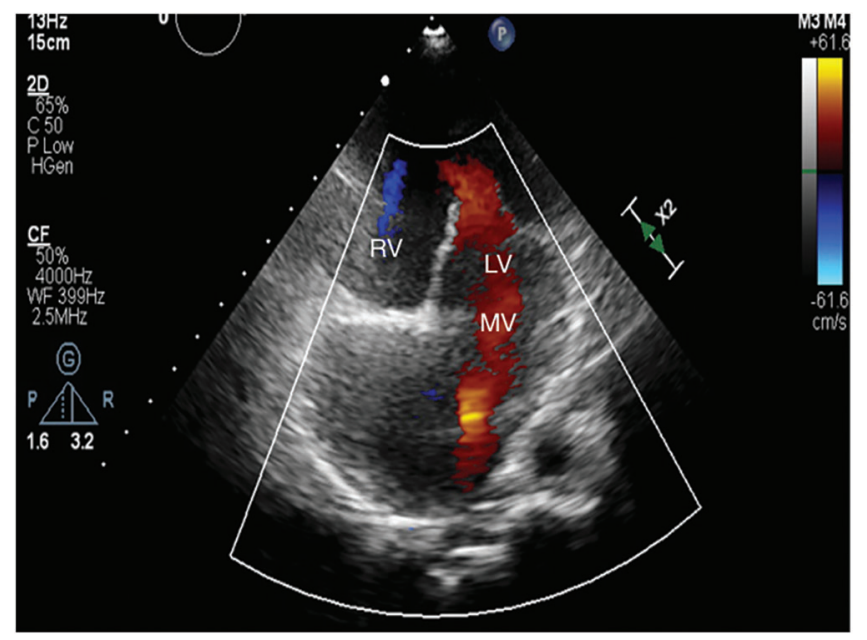

Figure 1: Apical 4 chamber view showing tricuspid atresia in patient number 2 (MV: mitral valve, LV: left ventricle, RV: right ventricle)

The morphology of the dominant ventricle (right, left or indeterminate), the location of the rudimentary ventricles or outlet chambers (anterior or posterior, left or right); the location of the papillary muscles and chordal attachments within the ventricular chambers (straddling and/or overriding) were assessed.

Assessment of dominant ventricular systolic function:

If the dominant ventricular morphology was left, then the ventricular function was assessed by M-mode or 2-dimensional echocardiography [7] and the modified Simpson biplane method [8]. 


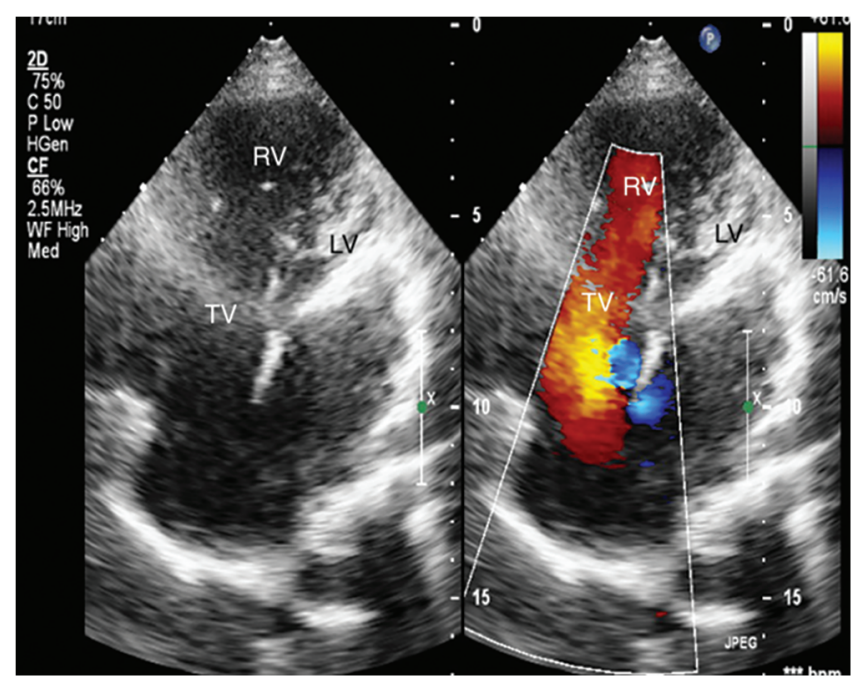

Figure 2: Apical 4 chamber view showing mitral atresia in patient number 15 (TV: Tricuspid valve, LV: left ventricle, RV: right ventricle)

If the dominant ventricular morphology was right, then the ventricular function was assessed by tricuspid annular plane systolic excursion (TAPSE) and the right ventricle fractional area change (FAC). RV FAC $<35 \%$ indicates RV systolic dysfunction [7,9].

Assessment of the atrioventricular valves was done as well and the degree of regurgitation was noted.

Evaluation of pulmonary valve morphology, degree of pulmonary stenosis and regurgitation if present. Level of stenosis and gradient across the valve was recorded. In patients with previous banding, gradient across the band and its site were evaluated.

Pulmonary arteries size, flow and the presence of peripheral stenosis were noted.

The BDG was evaluated from the suprasternal notch view, a BDG might be right, left sided or bilateral in patients with bilateral superior vena cava. Patent flow was assessed by the presence of laminar flow on color Doppler and with low phasic velocity flow pattern on pulsed-wave Doppler and respiratory variation was also assessed. A significant obstruction was determined by a measured mean gradient of more than $2 \mathrm{mmHg}$ between the BDG circuit and the pulmonary arteries (Fig. 3) [10].

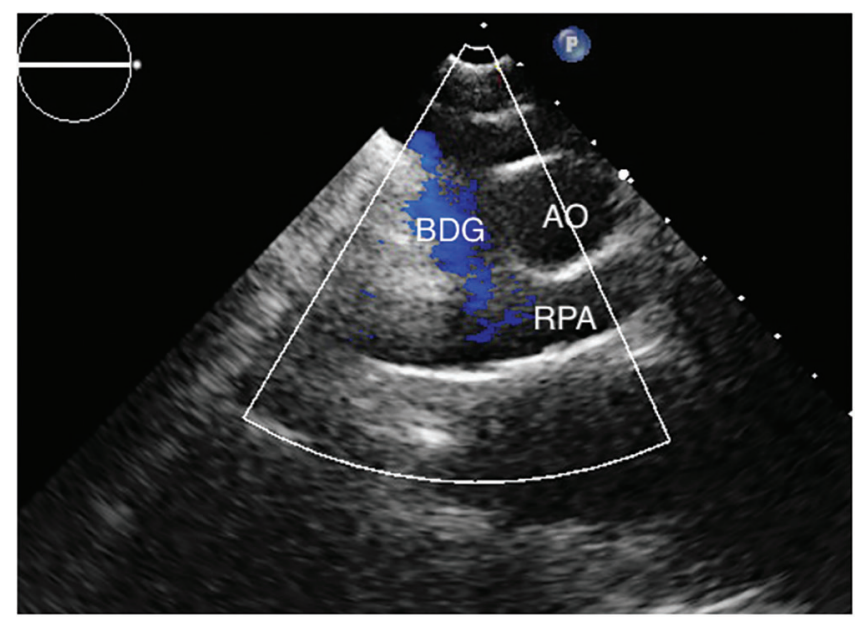

Figure 3: Suprasternal view with color flow showing patent right sided BDG with no reversal of flow in patient number 21 (BDG: bidirectional Glenn, AO: aorta, RPA: right pulmonary artery) 


\subsection{Cardiac Catheterization}

Cardiac catheterization was done to selected patients if Fontan completion was planned, hemodynamic assessment was needed due to desaturation or catheter-based interventions were required.

\subsubsection{Hemodynamic Study: Under GA and 100\% Oxygen}

Various pressures (aorta and pulmonary artery systolic, diastolic and mean pressures and dominant ventricle systolic and diastolic pressures) and saturations (aorta and pulmonary artery saturations) were recorded, veno-venous collaterals and aortopulmonary collaterals were looked for and assessment of peripheral pulmonary arteries for significant peripheral pulmonary stenosis was done.

\subsubsection{Percutaneous Interventions}

Percutaneous interventions were done to few patients and most commonly indicated for peripheral pulmonary stenting or collaterals closure (Fig. 4).

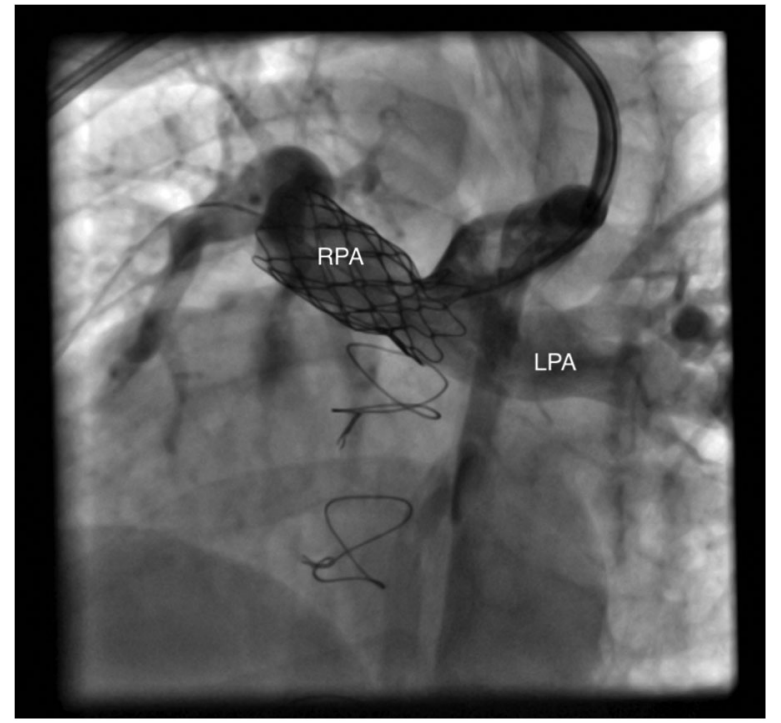

Figure 4: Showing well deployed RPA stent in patient number 10 (RPA: right pulmonary artery, LPA: left pulmonary artery)

\section{Statistical Analysis}

The collected data was summarized, presented and analyzed using the commercially available software (SPSS 19.0 version, IBM Co., Armonk, NY, USA). Qualitative data was summarized by frequency and percentages and quantitative data was expressed as mean \pm standard deviation. Chi square test was used to compare the systemic ventricle morphology with the degree of functional capacity limitation. The correlation between the RDW and the oxygen saturation was assessed by linear regression and Pearson correlation analysis. $P<0.05$ was considered statistically significant.

\section{Results}

\subsection{Demographic Data}

Primarily, our registry included 194 patients; however, 16 patients were excluded (4 were critically ill and 12 had refused the study) with remaining 178 patients. The mean age of the registry was $18.7 \pm$ 8.26 years (range between 5 and 37 years), 85 males (48\%) and 93 females (52\%). 
128 patients $(72 \%)$ were single, 46 patients $(26 \%)$ were married and 4 patients $(2 \%)$ were divorced. 17 females post BDG got pregnant and gave birth to healthy offspring.

In our study, 87 patients (49\%) had a systemic ventricle of right ventricular morphology, while, 91 patients $(51 \%)$ had a systemic ventricle of left ventricular morphology. The primary diagnosis had a wide range among the commonest were double outlet right ventricle with hypoplastic left ventricle and tricuspid atresia (Table 1).

Table 1: Primary diagnosis

\begin{tabular}{llll}
\hline & & Number & Percentage \\
\hline Ventricular morphology & Primary diagnosis & & \\
Dominant right ventricular & Double outlet right ventricle with hypoplastic & 45 & $25.3 \%$ \\
morphology & & \\
& left ventricle & 26 & $14.6 \%$ \\
& Mitral atresia & 10 & $5.6 \%$ \\
& Transposition of great arteries & 6 & $3.4 \%$ \\
& Unbalanced common atrio-ventricular canal & 6 & $21.3 \%$ \\
Dominant left ventricular & Tricuspid atresia & 38 & $14 \%$ \\
& Double inlet left ventricle & 25 & $6.7 \%$ \\
& Unbalanced common atrio-ventricular canal & 12 & $2.8 \%$ \\
& Tetralogy of Fallot & 5 & $2.3 \%$ \\
& Pulmonary atresia & 4 & $2.3 \%$ \\
& Hypoplastic right ventricle & 4 & $1.1 \%$ \\
& Ebstein anomaly & 2 & $0.6 \%$ \\
\hline
\end{tabular}

105 patients (59\%) had undergone only BDG, while 73 patients $(41 \%)$ had undergone surgeries prior to BDG. In addition to that, 42 patients (23.6\%) had undergone percutaneous intervention (Table 2).

Table 2: Surgical and percutaneous interventions

\begin{tabular}{lll}
\hline & Number & Percentage \\
\hline Surgical intervention prior to bidirectional Glenn shunt (BDG) & & \\
None & 105 & $59 \%$ \\
Pulmonary artery banding & 47 & $27 \%$ \\
Modified Blalock-Taussig shunt & 24 & $13 \%$ \\
Pulmonary artery banding and modified Blalock-Taussig shunt & 2 & $1 \%$ \\
Percutaneous intervention & & \\
None & 136 & $76.3 \%$ \\
Rashkind balloon atrial septostomy prior to BDG & 19 & $10.7 \%$ \\
Peripheral pulmonary stenting after BDG & 10 & $5.6 \%$ \\
Veno-venous collateral closure after BDG & 8 & $4.5 \%$ \\
Multiple hemodynamic studies after BDG & 3 & $1.7 \%$ \\
Balloon pulmonary valvuloplasty after BDG & 1 & $0.6 \%$ \\
Accessory pathway ablation after BDG & 1 & $0.6 \%$ \\
\hline
\end{tabular}


Patients underwent BDG at a median age of 6 years (range between 1 and 34 years) (Fig. 5).

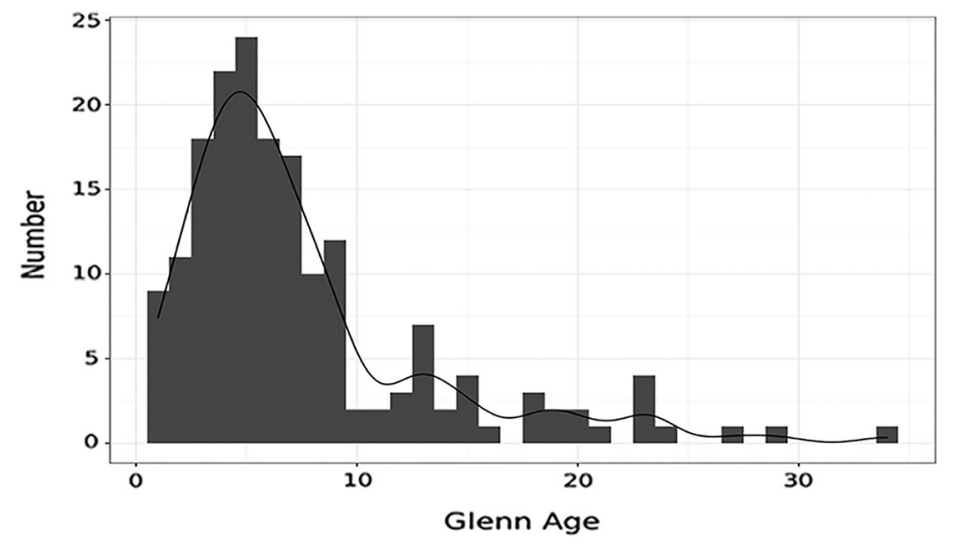

Figure 5: Showing age at which patients underwent BDG

Currently, 27 patients (15\%) had no limitations to daily physical activities and 151 patients (85\%) suffered from limitation to daily physical activity; among the sufferers, 78 patients (44\%) claimed to have mild limitation, 67 patients (38\%) complained of having moderate limitation and 6 patients (3\%) had severe limitation of daily physical activity (Fig. 6). In order to have a more objective evaluation of the functional capacity of our patients, the six-minutes-walk test was used. The distance walked during the test ranged between 108 and $504 \mathrm{~m}$ with a mean of $302 \pm 82.7 \mathrm{~m}$ with a mean decrease in oxygen saturation pre and post exercise of $7.56 \pm 6.07 \%$. However, the mean recovery time required to reach baseline oxygen saturation was $2.45 \pm 1.10 \mathrm{~min}$.

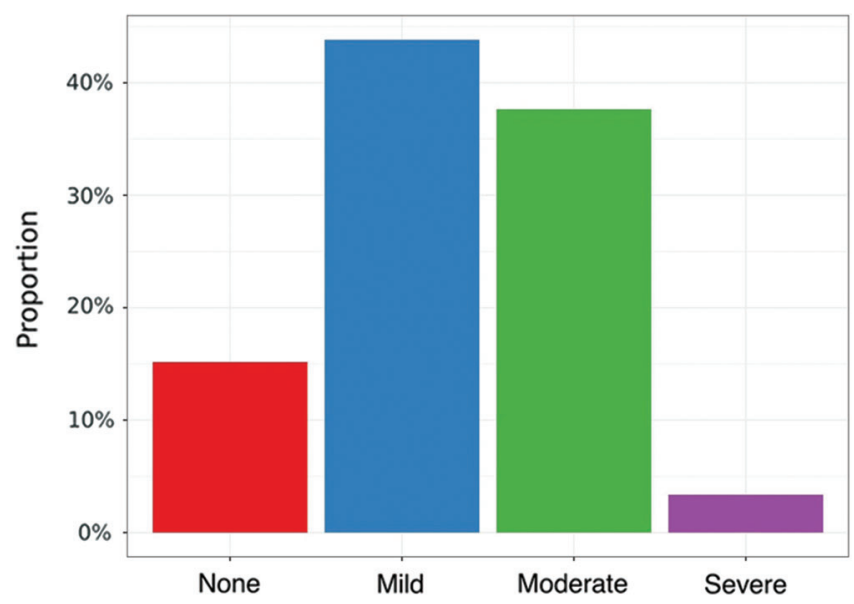

Figure 6: Showing the degree of physical activity limitation among patients

66 patients (37\%) take no medications at all; on the other hand, 112 patients $(63 \%)$ are on various medications [53 patients (30\%) are on aspirin therapy, 28 patients $(16 \%)$ are on iron therapy, 15 patients $(8 \%)$ are on anti-failure treatment, 9 patients $(5.1 \%)$ are on folic acid therapy, 4 patients $(2.2 \%)$ are on febuxostat treatment and 3 patients $(1.7 \%)$ are on warfarin]. 


\subsection{Six-Minutes-Walk Test}

The functional capacity of our patients was assessed by using the six-minute-walk test. The distance walked during the test ranged between 108 and $504 \mathrm{~m}$ with a mean of $302 \pm 82.7 \mathrm{~m}$ with a mean decrease in oxygen saturation pre and post exercise of $7.56 \pm 6.07 \%$. However, the mean recovery time required to reach baseline oxygen saturation was $2.45 \pm 1.10 \mathrm{~min}$.

\subsection{Laboratory Findings}

It was noted that most of our cohort had polycythemia, however, the blood indices showed microcytic hypochromic pattern denoting the presence of iron deficiency anemia. The hemoglobin ranged from 10 to $24 \mathrm{~g} / \mathrm{dl}$ with a mean of $16.7 \pm 2.48 \mathrm{~g} / \mathrm{dl}$ and the hematocrit value ranged from 22 to $73 \%$ with a mean of $60.8 \pm 7.27 \%$. The mean RBC count was $7.61 \pm 1.45$ million $/ \mathrm{cmm}$, the mean MCV was $76.8 \pm 10.3 \mathrm{fl}$, the mean $\mathrm{MCH}$ was $25.1 \pm 3.85 \mathrm{pg}$, the mean $\mathrm{MCHC}$ was $31.7 \pm 2.01 \mathrm{~g} / \mathrm{dl}$ and the RDW ranged from 11.8 to $32.3 \%$ with a mean of $21.3 \pm 4.20 \%$. Rest of laboratory findings are summarized in Table 3 .

Table 3: Laboratory findings

\begin{tabular}{lll}
\hline & Mean \pm SD & Range \\
\hline Hemoglobin & $16.7 \pm 2.48$ & $10-24$ \\
Hematocrit value & $60.8 \pm 7.27$ & $22-73$ \\
RBC count & $7.61 \pm 1.45$ & $4.79-11.3$ \\
MCV & $76.8 \pm 10.3$ & $46.6-99$ \\
MCH & $25.1 \pm 3.85$ & $13.7-33.2$ \\
MCHC & $31.7 \pm 2.01$ & $25.8-36.4$ \\
RDW & $21.3 \pm 4.20$ & $11.8-32.3$ \\
TLC & $6.81 \pm 1.86$ & $3-15.9$ \\
PLT & $191 \pm 48.8$ & $86-329$ \\
Urea & $30.8 \pm 6.95$ & $10-81$ \\
Creatinine & $0.743 \pm 0.172$ & $0.4-1.32$ \\
Uric acid & $7.26 \pm 2.12$ & $3.1-16$ \\
Sodium & $141 \pm 2.76$ & $128-149$ \\
Potassium & $4.61 \pm 0.534$ & $3.4-6.4$ \\
AST & $19 \pm 4.45$ & $10-52$ \\
ALT & $14.3 \pm 4.42$ & $3-29$ \\
Total bilirubin & $0.788 \pm 0.253$ & $0.39-2.1$ \\
Direct bilirubin & $0.257 \pm 0.09$ & $0.03-0.7$ \\
INR & $1.15 \pm 0.233$ & $0.8-2.9$ \\
\hline & &
\end{tabular}

\subsection{Echocardiographic Findings}

In our 91 patients with left ventricular morphology, the mean LV end diastolic volume was $169 \pm$ $11.4 \mathrm{ml}$, the mean LV end systolic volume was $79 \pm 8.42 \mathrm{ml}$ and the LV ejection fraction ranged between $34.3 \%$ and $67.2 \%$ with a mean of $53.3 \pm 3.29 \%$. In our 87 patients with right ventricular morphology, the mean RV end diastolic area was $37.6 \pm 4.62 \mathrm{~cm}^{2}$, the mean RV end systolic area was $22.1 \pm 3.62 \mathrm{~cm}^{2}$ and the RV fractional area change ranged between $20 \%$ and $58.7 \%$ with a mean of $41.1 \pm 5.47 \%$. The atrio- 
ventricular valvular findings together with the pulmonary valve and peripheral pulmonary branches assessment results are shown in Table 4.

Table 4: Atrio-ventricular valves, pulmonary valve and peripheral pulmonary branches assessment

\begin{tabular}{|c|c|c|}
\hline & Number & Percentage \\
\hline \multicolumn{3}{|l|}{ Left atrio-ventricular valve } \\
\hline Competent & 30 & $17 \%$ \\
\hline Trivial regurgitation & 28 & $16 \%$ \\
\hline Mild regurgitation & 66 & $37 \%$ \\
\hline Moderate regurgitation & 24 & $13 \%$ \\
\hline Severe regurgitation & 4 & $2 \%$ \\
\hline Atretic & 26 & $15 \%$ \\
\hline \multicolumn{3}{|l|}{ Right atrio-ventricular valve } \\
\hline Competent & 12 & $6.7 \%$ \\
\hline Trivial regurgitation & 31 & $17.4 \%$ \\
\hline Mild regurgitation & 55 & $30.9 \%$ \\
\hline Moderate regurgitation & 30 & $16.9 \%$ \\
\hline Severe regurgitation & 12 & $6.7 \%$ \\
\hline Atretic & 38 & $21.4 \%$ \\
\hline \multicolumn{3}{|l|}{ Pulmonary valve } \\
\hline Native stenosis & 112 & $62.9 \%$ \\
\hline Atretic & 17 & $9.6 \%$ \\
\hline Pulmonary artery band & 49 & $27.5 \%$ \\
\hline \multicolumn{3}{|l|}{ Peripheral pulmonary branches size } \\
\hline Good & 176 & $99 \%$ \\
\hline Small & 2 & $1 \%$ \\
\hline \multicolumn{3}{|l|}{ Peripheral pulmonary branches stenosis } \\
\hline Absent & 162 & $91 \%$ \\
\hline Left pulmonary artery stenosis & 10 & $5.6 \%$ \\
\hline \multirow[t]{2}{*}{ Right pulmonary artery stenosis } & 6 & $3.4 \%$ \\
\hline & Mean $\pm \mathrm{SD}$ & Range \\
\hline Peak pressure gradient across pulmonary valve & $78.4 \pm 11$ & $50-102 \mathrm{mmHg}$ \\
\hline
\end{tabular}

During Glenn shunt assessment, 156 patients (88\%) were found to have a right sided Glenn, 8 patients (4\%) were found to have a left sided Glenn and 14 patients $(8 \%)$ were found to have bilateral Glenn. Significant flow reversal was present in 24 patients (13\%) and mean Glenn shunt mean pressure gradient was $1.07 \pm 0.29 \mathrm{mmHg}$. 


\subsection{Cardiac Catheterization Findings}

In 154 patients who underwent hemodynamic cardiac catheterization, the mean pulmonary artery pressure had a mean of $12 \pm 5.29 \mathrm{mmHg}$ and the mean aortic artery pressure had a mean of $82.7 \pm$ $12.4 \mathrm{mmHg}$. The dominant ventricle mean diastolic pressure was $11.2 \pm 3.03 \mathrm{mmHg}$. The mean invasive systemic oxygen saturation was $84.7 \pm 7.57 \%$. Added to that, 14 patients $(9 \%)$ were found to have significant proximal peripheral pulmonary artery branches stenosis [9 patients $(5.8 \%)$ having left pulmonary artery stenosis and 5 patients $(3.2 \%)$ having right pulmonary artery stenosis]. Moreover, 90 patients $(58 \%)$ had significant veno-venous collaterals, 74 patients $(48 \%)$ had minor aorto-pulmonary collaterals, 23 patients (15\%) had major aorto-pulmonary collaterals and 10 patients (6.5\%) had significant AV malformations.

\subsection{Important Correlation}

\subsubsection{Effect of Dominant Ventricular Morphology on Degree of Limitation of Daily Physical Activity}

In our study, there was no statistically significant difference between dominant ventricle morphology and degree of limitation of daily physical functioning.

In 91 patients with left ventricular morphology, 12 patients (13\%) had no limitations on daily physical activity, 40 patients (44\%) had mild limitations on daily physical activity, 35 patients $(38 \%)$ had moderate limitations on daily physical activity and 4 patient $(5 \%)$ had severe limitations on daily physical activity.

In 87 patients with right ventricular morphology, 15 patients (17\%) had no limitations on daily physical activity, 38 patients (43.5\%) had mild limitations on daily physical activity, 32 patients (37\%) had moderate limitations on daily physical activity and 2 patient $(2.5 \%)$ had severe limitations on daily physical activity (Table 5).

Table 5: Effect of dominant ventricular morphology on degree of limitation of daily physical activity

\begin{tabular}{llllll}
\hline \multicolumn{5}{c}{ Degree of limitation of daily physical functioning } \\
\hline Dominant ventricle & None & Mild & Moderate & Severe & \\
\hline Left & 12 & 40 & 35 & 4 & $91(51.1 \%)$ \\
& $13.2 \% \mathrm{RT}$ & $44.0 \% \mathrm{RT}$ & $38.5 \% \mathrm{RT}$ & $4.4 \% \mathrm{RT}$ & \\
& $44.4 \% \mathrm{CT}$ & $51.3 \% \mathrm{CT}$ & $52.2 \% \mathrm{CT}$ & $66.7 \% \mathrm{CT}$ & \\
& $6.7 \% \mathrm{GT}$ & $22.5 \% \mathrm{GT}$ & $19.7 \% \mathrm{GT}$ & $2.2 \% \mathrm{GT}$ & \\
Right & 15 & 38 & 32 & 2 & $87(48.9 \%)$ \\
& $17.2 \% \mathrm{RT}$ & $43.7 \% \mathrm{RT}$ & $36.8 \% \mathrm{RT}$ & $2.3 \% \mathrm{RT}$ & \\
& $55.6 \% \mathrm{CT}$ & $48.7 \% \mathrm{CT}$ & $47.8 \% \mathrm{CT}$ & $33.3 \% \mathrm{CT}$ & \\
& $8.4 \% \mathrm{GT}$ & $21.3 \% \mathrm{GT}$ & $18.0 \% \mathrm{GT}$ & $1.1 \% \mathrm{GT}$ & \\
& 27 & 78 & 67 & 6 & 178 \\
& $(15.2 \%)$ & $(43.8 \%)$ & $(37.6 \%)$ & $(3.4 \%)$ & \\
Significance level & $P=0.7780$ & & & & \\
\hline
\end{tabular}

\subsubsection{Effect of Veno-Venous Collaterals Presence and Degree of Desaturation During the Six-Minutes-Walk Test}

In our study, there was statistically significant difference between patients with and without veno-venous collateral presence detected by catheterization regarding the degree of desaturation evaluated during the sixminutes-walk test. 
The mean oxygen desaturation among the patients having veno-venous collateral was $8.6 \pm 6.8 \%$; in contrast to a mean oxygen desaturation of $6.1 \pm 4.6 \%$ among the patients having no veno-venous collaterals $(P=0.008)$.

\subsubsection{Effect of RDW on Oxygen Saturation}

In our study, there was statistically significant difference between patients with high oxygen saturation (above $80 \%$ ) and patients with low oxygen saturation (below $80 \%$ ) regarding the RDW.

The mean RDW among the patients with high oxygen saturation (above $80 \%$ ) was $20.7 \pm 4.18 \%$; in contrast to a mean RDW of $22.3 \pm 4.05 \%$ among the patients with low oxygen saturation (below $80 \%$ ) $(P=0.012)$.

Using ROC curve analysis; a RDW cutoff value was generated to predict lowered oxygen saturation. The area under this ROC curve was 0.624 and $P$-value was 0.004 . A RDW cutoff value of $21.8 \%$ provided the highest balanced sensitivity $(53.85 \%)$ and specificity $(69.91 \%)$ to determine oxygen saturation less than $80 \%$ on room air. A cutoff value more than $32.3 \%$ had $100 \%$ specificity to predict oxygen saturation less than $80 \%$ on room air (Fig. 7 ).

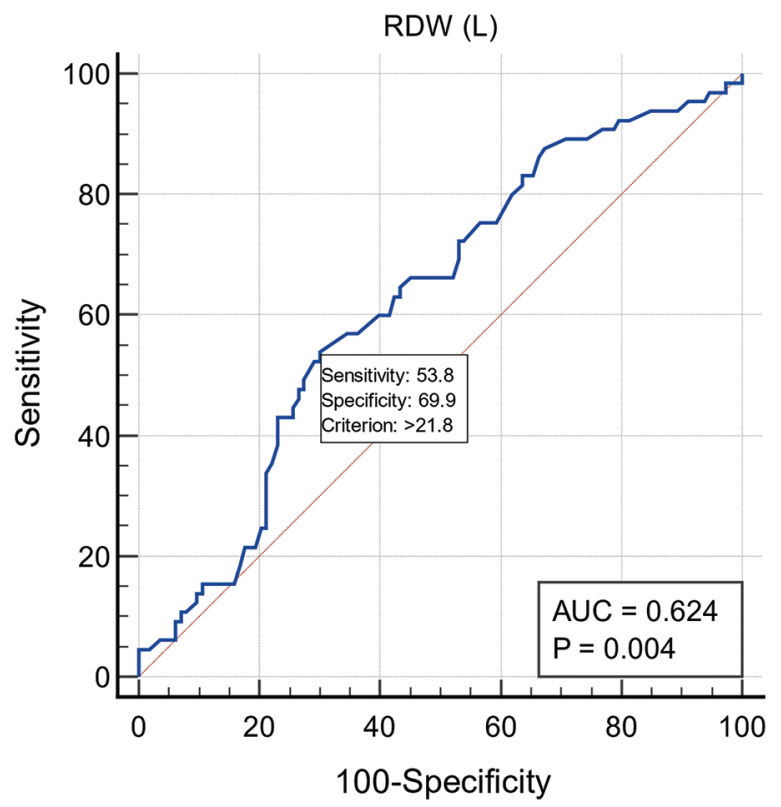

Figure 7: ROC curve showing a RDW cutoff value of $21.8 \%$ provided the best balanced sensitivity $53.85 \%$ and specificity $69.91 \%$ to determine oxygen saturation less than $80 \%$

\subsection{Long Term Outcome}

A sub-analysis was done in our study demonstrating 87 patients who had BDG aged more than 10 years. In this cohort of patients, 41 males (47\%) and 46 females (53\%) with a mean age of 23.1 years.

The most common primary diagnosis was tricuspid atresia (28\%). Prior to BDG, 23 patients had undergone pulmonary artery band, 16 patients had MBT shunt, 1 patient had PAB + MBT shunt and 12 patients had undergone Rashkind balloon atrial septostomy. While after BDG procedure, 8 patients had undergone veno-venous collaterals closure and 5 patients had peripheral pulmonary artery stenting. 
Although their invasive systemic oxygen saturation was $84.2 \%$, those patients had an acceptable functional capacity as only 25 patients $(28 \%)$ claimed NYHA class III and 3 patients $(3.5 \%)$ had NYHA class IV. While tested by 6MWT, the mean walked distance was 295 meters.

Their blood indices showed polycythemia with the same pattern of the microcytic hypochromic iron deficiency anemia as our main cohort. The mean hemoglobin was $17.2 \mathrm{gm} / \mathrm{dl}$, hematocrit was $59.6 \mathrm{gm} / \mathrm{dl}$, MCV was $76.2 \mathrm{fl}, \mathrm{MCH}$ was $24.7 \mathrm{pg}$ and RDW was $21.8 \%$.

During cardiac catheterization, their mean pulmonary artery pressure had a mean of $12.4 \mathrm{mmHg}$ and the mean dominant ventricle diastolic pressure was $11.4 \mathrm{mmHg}$. 9 patients $(10 \%)$ had evidence of peripheral pulmonary artery stenosis, 61 patients $(70 \%)$ had veno-venous collaterals, 8 patients $(9 \%)$ had major significant aorto-pulmonary collaterals and only 7 patients $(8 \%)$ had AV malformations.

\section{Discussion}

It is well known that Fontan procedure is the final step in single ventricle palliation. However, in our country and due to late presentation and unavailability of heart-lung transplantation, BDG was considered the preferred palliative step. As it carries a lower rate of complications compared to Fontan procedure.

In our study, we tried to demonstrate the actual outcomes following BDG as well as our patients' functional capacity and comorbidities. These data were also highlighted in our sub-analysis of 10 and more years following BDG patients.

In our study, there was no statistically correlation between the basic anatomy nor the dominant ventricular morphology and our patients' functional capacity. Similarly, Sen et al. [11] noted that neither the basic anatomy nor the dominant ventricular morphology could affect the distances walked during the six-minutes-walk test among his patients. He correlated the distances walked during the six-minutes-walk test with the resting heart rate [11]. On the opposite side, Alsoufi et al. [12] studied 227 patients and concluded that the prognosis of single ventricle patients is greatly affected by underlying cardiac anatomy and type of dominant ventricle, as patients with dominant left ventricular morphology can better accommodate to volume and pressure overload and develop late systolic or diastolic disfunction. He also observed that patients with tricuspid atresia and double inlet left ventricle had the best prognosis [12].

In our study, 73 patients (41\%) had undergone prior surgeries that included pulmonary artery banding, MBT shunt and combined pulmonary artery banding with MBT shunt. Compared to a study conducted by Yamada et al. [13], 34 patients were included in the study. 6 patients (18\%) had undergone BDG only, while 12 patients $(35 \%)$ had undergone a single MBT shunt, 6 patients $(17 \%)$ had undergone two MBT shunts and 10 patients (30\%) had undergone pulmonary artery banding [13].

Our patients who underwent BDG had a median age of 6 years ranging between 1 and 34 years; similarly in the study of Tariq et al. in Pakistan (a developing country like ours), the mean age at which their 79 patients underwent BDG was 4.5 years [14]. Added to that, Sen et al. [11], who conducted his study in India, demonstrated that the mean age at BDG was 7.5 years \pm 5.1 years [11]. This age was higher than the actual minimum age for BDG and it might be due to late presentation and delayed seeking medical advice. Hence, the hypoplastic left heart identity was not represented in either study possibly due to delayed diagnoses and death before Norwood operation. In addition, Al-Dairy et al. [14] who studied 161 patients in Iran, found that the median age at which his patients underwent BDG was $5 \pm 4.9$ years ranging between 9 months and 24.5 years [15].

The relative older age of children undergoing the operation in developing countries is due to the late presentation, delayed diagnosis and lack of primary health care facilities. Added to that, insufficient awareness regarding congenital heart disease and poor socio-economic status played a huge role in delaying the age at which operation should be done. 
Regarding the degree of limitation of daily physical activities of our 178 patients, we had identified 27 patients $(15 \%)$ with no limitations at all, 78 patients $(44 \%)$ with mild limitations, 76 patients $(38 \%)$ with moderate limitations and 6 patients $(3 \%)$ with severe degree of limitation. Comparatively with the evaluation of the degree of exercise tolerance done by Yamada et al. [13], on 34 patients, 11.1\% of the patients had normal exercise tolerance, $40.7 \%$ of the patients had mild exercise limitation, $48.2 \%$ of the patients had moderate exercise limitation and no patients had severe exercise limitations [13]. This difference could be due to the few numbers of patients studied compared to our registry.

In addition, a six-minutes-walk test was performed to further assess functional capacity of our 178 patients and it was found that the mean distance walked during the test was $302 \mathrm{~m} \pm 82.7 \mathrm{~m}$ with mean of degree of desaturation was $7.56 \% \pm 6.07 \%$. However, Sen et al. [11] had evaluated 44 patients post BDG by six-minutes-walk test and it was found that the mean distance walked was $374 \mathrm{~m} \pm 83 \mathrm{~m}$ with mean of degree of desaturation was $21.8 \% \pm 19.7 \%$ [11].

A probable cause of decreased functional capacity noted in our patients especially the more aged patients with BDG operation performed for 10 or more years was the relative iron deficiency anemia causing a microcystic hypochromic pattern. This pattern was noted in the majority of patients with mean MCV of $76.8 \pm 10.3 \mathrm{fl}$ and $\mathrm{MCH}$ of $25.1 \pm 3.85 \mathrm{pg}$. However only 28 patients $(16 \%)$ were on iron therapy. Thus, we believe that prompt iron therapy to our patients could result in better quality of life.

In addition to that, another cause for significant desaturation during six-minutes-walk test was the presence of veno-venous collaterals, 90 patients (58\%) were found to have significant veno-venous collaterals, this percentage had risen to $70 \%$ in our cohort with 10 or more years BDG. Nevertheless, 8 patients underwent veno-venous collateral closure. Among the group of patients with veno-venous collaterals, we have noted significantly higher degree of oxygen desaturation during the six-minutes-walk test.

This was supported by a study on 54 patients conducted by McElhinney et al. [15], who found that 18 patients (33\%) had veno-venous collaterals and 6 patients had undergone coil embolization for venovenous collateral closure. Those 18 patients had a significantly lower systemic oxygen saturation [16].

Additional reason of systemic oxygen desaturation is the development of arterio-venous malformation. Within our 154 patients who were investigated by cardiac catheterization, 10 patients $(6.5 \%)$ were found to have arterio-venous malformation. However, Kim et al. [17] had studied 27 patients with BDG and only 4 patients $(14 \%)$ had typical arterio-venous malformation during pulmonary angiography [17]. The most common theories behind pulmonary arterio-venous malformation development are the lack of hepatic venous effluent directly perfusing the pulmonary arteries or the loss of pulsatile pulmonary blood flow [18].

\section{Conclusion}

The follow up of our post BDG patients had demonstrated some unique features including an older age while undergoing the procedure and an acceptable function capacity although slight systemic desaturation. This functional capacity can be improved by treatment of the relative iron deficiency anemia and timely catheter-based interventions like collaterals closure and peripheral pulmonary stenting. Thus, in developing country like ours where the rate of complication of Fontan procedure is relatively high and the absence of heart-lung transplant program, BDG can be considered a safe and acceptable palliation for single ventricle patients.

\section{Study Limitations}

It was a single center study; however, the sample size was relatively larger compared with previous studies on patients follow up after BDG. Another limitation of our study was the lack of a post Fontan cohort that could allow a more comparison between post BDG and post Fontan outcomes; thus, another 
comparative study evaluating post BDG and post Fontan patients will be conducted. Due to limited resources, iron profile was unavailable for our patients.

Ethics Approval: The study was approved by Research Ethics Committee at the Faculty of Medicine Ain Shams University (FWA 000017585). Approval No. FMASU M D 65/2019.

Availability of Data and Materials: The data sets used and analyzed during the present study are available upon request.

Funding Statement: This research did not receive any specific grant from funding agencies in the public, commercial, or not-for-profit sectors.

Conflicts of Interest: The authors declare that they have no conflicts of interest to report regarding the present study.

\section{References}

1. Pridjian, A. K., Mendelsohn, A. M., Lupinetti, F. M., Beekman, R. H. III, Dick, M. II et al. (1993). Usefulness of the bidirectional Glenn procedure as staged reconstruction for the functional single ventricle. The American Journal of Cardiology, 71(11), 959-962. DOI 10.1016/0002-9149(93)90914-X.

2. Dixit, R., Rai, S. K., Yadav, A. K., Lakhotia, S., Agrawal, D. et al. (2015). Epidemiology of congenital heart disease in India. Congenital Heart Disease, 10(5), 437-446. DOI 10.1111/chd.12220.

3. Freedom, R. M., Nykanen, D., Benson, L. N. (1998). The physiology of the bidirectional cavopulmonary connection. The Annals of Thoracic Surgery, 66(2), 664-667. DOI 10.1016/S0003-4975(98)00618-3.

4. Kouchoukos, N. T., Blackstone, E. H., Hanley, F. L., Kirklin, J. K. (2013). Tricuspid atresia and single ventricle physiology. In: Kirklin, J. K., Barratt-Boyes, B. G. (Eds.), Cardiac surgery: Morphology, diagnostic criteria, natural history, techniques, results, and indications, pp. 1509-1574. Philadelphia, PA: Saunders.

5. Donofrio, M. T., Jacobs, M. L., Spray, T. L., Rychik, J. (1998). Acute changes in preload, afterload, and systolic function after superior cavopulmonary connection. The Annals of Thoracic Surgery, 65(2), 503-508. DOI 10.1016/S0003-4975(97)00866-7.

6. ATS Committee on Proficiency Standards for Clinical Pulmonary Function Laboratories (2002). ATS statement: Guidelines for the six-minute walk test. American Journal of Respiratory and Critical Care Medicine, 166(1), 111-117. DOI 10.1164/ajrccm.166.1.at1102.

7. Lang, R. M., Badano, L. P., Mor-Avi, V., Afilalo, J., Armstrong, A. et al. (2015). Recommendations for cardiac chamber quantification by echocardiography in adults: An update from the American Society of Echocardiography and the European Association of Cardiovascular Imaging. Journal of the American Society of Echocardiography: Official Publication of the American Society of Echocardiography, 28(1), 1-39.e14.

8. Lang, R. M., Bierig, M., Devereux, R. B., Flachskampf, F. A., Foster, E. et al. (2005). Chamber Quantification Writing Group, American Society of Echocardiography's Guidelines and Standards Committee, \& European Association of Echocardiography. Recommendations for chamber quantification: A report from the American Society of Echocardiography's Guidelines and Standards Committee and the Chamber Quantification Writing Group, developed in conjunction with the European Association of Echocardiography, a branch of the European Society of Cardiology. Journal of the American Society of Echocardiography: Official Publication of the American Society of Echocardiography, 18(12), 1440-1463.

9. Rudski, L. G., Lai, W. W., Afilalo, J., Hua, L., Handschumacher, M. D. et al. (2010). Guidelines for the echocardiographic assessment of the right heart in adults: A report from the American Society of Echocardiography endorsed by the European Association of Echocardiography, a registered branch of the European Society of Cardiology, and the Canadian Society of Echocardiography. Journal of the American Society of Echocardiography, 23(7), 685-713. DOI 10.1016/j.echo.2010.05.010.

10. Gewillig, M., Mertens, L. L. (2016). Echocardiographic assessment of functionally single ventricles after the Fontan Operation. In: Echocardiography in pediatric and congenital heart disease: from fetus to adult. 2nd edition. pp. 541-557. West Sussex, UK: Willey Blackwell. 
11. Sen, S., Bandyopadhyay, B., Eriksson, P., Chattopadhyay, A. (2012). Functional capacity following univentricular repair-midterm outcome. Congenital Heart Disease, 7(5), 423-432.

12. Alsoufi, B., Manlhiot, C., Awan, A., Alfadley, F., Al-Ahmadi, M. et al. (2012). Current outcomes of the Glenn bidirectional cavopulmonary connection for single ventricle palliation. European Journal of Cardio-Thoracic Surgery: Official Journal of the European Association for Cardio-Thoracic Surgery, 42(1), 42-49.

13. Yamada, K., Roques, X., Elia, N., Laborde, M. N., Jimenez, M. et al. (2000). The short- and mid-term results of bidirectional cavopulmonary shunt with additional source of pulmonary blood flow as definitive palliation for the functional single ventricular heart. European Journal of Cardio-Thoracic Surgery: Official Journal of the European Association for Cardio-Thoracic Surgery, 18(6), 683-689. DOI 10.1016/S1010-7940(00)00583-2.

14. Tariq, M, Zahid, I, Hashmi, S, Amanullah, M, Shahabuddin, S. (2021). The Glenn procedure: Clinical outcomes in patients with congenital heart disease in pakistan. Annals of cardiac anaesthesia, 24(1), 30-35.

15. Al-Dairy, A., Dehaki, M. G., Omrani, G., Sadeghpour, A., Jalali, A. H. et al. (2017). The outcomes of superior cavopulmonary connection operation: A single center experience. Brazilian Journal of Cardiovascular Surgery, 32(6), 503-507. DOI 10.21470/1678-9741-2017-0025.

16. McElhinney, D. B., Reddy, V. M., Hanley, F. L., Moore, P. (1997). Systemic venous collateral channels causing desaturation after bidirectional cavopulmonary anastomosis: Evaluation and management. Journal of the American College of Cardiology, 30(3), 817-824. DOI 10.1016/S0735-1097(97)00223-4.

17. Kim, S. J., Bae, E. J., Cho, D. J., Park, I. S., Kim, Y. M. et al. (2000). Development of pulmonary arteriovenous fistulas after bidirectional cavopulmonary shunt. The Annals of Thoracic Surgery, 70(6), 1918-1922. DOI 10.1016/S0003-4975(00)02164-0.

18. Kavarana, M. N., Jones, J. A., Stroud, R. E., Bradley, S. M., Ikonomidis, J. S. et al. (2014). Pulmonary arteriovenous malformations after the superior cavopulmonary shunt: Mechanisms and clinical implications. Expert Review of Cardiovascular Therapy, 12(6), 703-713. DOI 10.1586/14779072.2014.912132. 